\title{
Spectral properties of reduced fermionic density operators and parity superselection rule
}

\author{
Grigori G. Amosov $1, *$ and Sergey N. Filippov $2,3,4, \dagger$ \\ ${ }^{1}$ V. A. Steklov Mathematical Institute, Russian Academy of Sciences, Gubkina Str. 8, Moscow, 119991, Russia \\ ${ }^{2}$ Institute of Physics and Technology, Russian Academy of Sciences, Nakhimovskii Pr. 34, Moscow 117218, Russia \\ ${ }^{3}$ Moscow Institute of Physics and Technology, Institutskii Per. 9, Dolgoprudny, Moscow Region 141700, Russia \\ ${ }^{4}$ P. N. Lebedev Physical Institute, Russian Academy of Sciences, Leninskii Pr. 53, Moscow 119991, Russia
}

\begin{abstract}
We consider pure fermionic states with a varying number of quasiparticles and analyze two types of reduced density operators: one is obtained via tracing out modes, the other is obtained via tracing out particles. We demonstrate that spectra of mode-reduced states are not identical in general and fully characterize pure states with equispectral mode-reduced states. Such states are related via local unitary operations with states satisfying the parity superselection rule. Thus, valid purifications for fermionic density operators are found. To get particle-reduced operators for a general system, we introduce the operation $\Phi(\varrho)=\sum_{i} a_{i} \varrho a_{i}^{\dagger}$. We conjecture that spectra of $\Phi^{p}(\varrho)$ and conventional $p$-particle reduced density matrix $\varrho_{p}$ coincide. Nontrivial generalized Pauli constraints are derived for states satisfying the parity superselection rule.
\end{abstract}

PACS numbers: 03.65.Aa, 03.65.Fd, 03.67.Bg, 05.30.Fk

\section{INTRODUCTION}

The physical nature of information [1] not only provides new ways of information processing (like quantum computing [2]) but also stimulates the research of specific information carriers - particles and quasiparticles with peculiar properties. This paper deals with particular quantum states which represent a superposition of states with different numbers of fermionic quasiparticles.

After electrons were argued to possess the additional degree of freedom [3] (later called spin [4]) and obey the Pauli exclusion principle [5], the Fermi-Dirac statistics was found [6, 7] and the general notion of fermion particles emerged. The antisymmetric nature of fermionic wavefunction [7] has provoked development of the canonical anticommutation relation [8] and the general theory of second quantization [9], which deals with systems with a varying number of particles. The fermionic statistics of half-integer spin particles was established in [10, 11]. Since every fermion has a half-integer spin, the total spin of an even number of fermions must be integer, whereas that of an odd number of fermions must be half-integer. Given a system with a varying number of particles, it is therefore possible to have a superposition of half-integer and integer total spins. However, it was recognized later that the comparison of phases between states with halfinteger and integer angular momenta cannot be reconciled with the requirement of relativistic invariance [12], which resulted in the formulation of parity (spinor, univalence) superselection rule (see, e.g., the reviews [13 15]). Any superselection rule relies on a group of physical transformations, for example the parity superselection rule relies on the rotational invariance [16] whereas the mass superselection rule relies on the Galilean invariance of nonrelativistic quantum mechanics [17]. If a system of leptons were invariant with respect to fermionic $U(1)$ transformations, there would be a leptonic family number su-

*Electronic address: gramos@mi.ras.ru

${ }^{\dagger}$ Electronic address: sergey.filippov@phystech.edu perselection rule, however, the recent experiments clearly indicate the neutrino oscillations (see, e.g., [18]), which contradicts to the conservation of leptonic family number (the violation was predicted in Ref. [19]). Thus, the experimental investigations of nature modify our understanding of fundamental symmetries, the physical models, and the mathematical frameworks used for their description. Recent experiments with massless Weyl fermionic quasiparticles [20 22] stimulate us to investigate properties of fermionic states in coherent superpositions of different number states. Moreover, superpositions of different number states usually emerge in fermionized models (see, e.g. [23]).

In this paper, we analyze properties of general pure fermionic states with a varying number of quasiparticles from the viewpoint of quantum information theory, namely, we consider the spectra of two reduced operators obtained from such a state by two different approaches: reduction of modes and reduction of particles.

Suppose a state space $H$ composed of two sets of modes $H_{1}$ and $H_{2}$. The mode represents a single-particle state that is either occupied or not. In quantum information theory, $H_{1}$ and $H_{2}$ may correspond to distant laboratories. In solid state physics, $H_{1}$ and $H_{2}$ may describe different positions or momenta of quasiparticles. In quantum chemistry, $H_{1}$ and $H_{2}$ may be attributed to two distinct sets of spin-orbits. In this paper, we consider situations, when the number of particles may change not only in $H_{1}$ and $H_{2}$ but in the common space $H$ too. Suppose a local physical observable $A_{1} \in \mathcal{A}_{1}$ acting on modes $H_{1}$ only, for instance, $A_{1}$ can be the local energy or the population of modes in $H_{1}$. On the one side, the quantummechanical mean value $\left\langle A_{1}\right\rangle=\omega\left(A_{1}\right)=\operatorname{Tr}\left(\varrho A_{1}\right)$, where $\omega$ is a functional defined on the common algebra of operators $\mathcal{A}=\mathcal{A}_{1} \times \mathcal{A}_{2}$ and $\varrho$ is the total density operator. On the other side, $\left\langle A_{1}\right\rangle=\omega_{1}\left(A_{1}\right)=\operatorname{Tr}\left(\varrho_{1} A_{1}\right)$, where $\omega_{1}$ is a functional defined on the algebra of local operators $\mathcal{A}_{1}$ and $\varrho_{1}$ is the mode-reduced density operator. [65] Analogously, $\omega_{2}$ and $\varrho_{2}$ represent a mode-reduced state of the second set of modes. Thus, for physical systems with two separate sets of single-particle sets, mode reduced states naturally occur as algebraic constructions. Such algebraic 
constructions are well defined for systems with a variable number of quasiparticles, so we explore spectral properties of $\omega_{1}$ and $\omega_{2}$ in this paper.

The inverse procedure to reduction is known as a purification and takes a simple form in tensor product spaces because the two spectra are identical (an immediate consequence of the Schmidt decomposition). We demonstrate that spectra of mode-reduced states do not necessarily coincide for a general fermionic state, which can be interpreted as a violation [24] of the Araki-Lieb inequality 25]. This fact should be taken into account when the entanglement of pure states is quantified via the entropy of one of subsystems [26]. The discrepancy between spectra (entropies) of subsystems of a pure state seems to be unphysical (since the result depends on labelling), so we further find necessary and sufficient conditions for spectra to be coincident for mode-reduced states. In particular, we not only find that spectra coincide for states satisfying the parity superselection rule [27] but also prove that all states with equispectral subsystems are the univalent states subjected to local unitary transformations.

Suppose now that one is interested in an average $p$ particle property, for instance, the average spin projection $(p=1)$ or the average two-electron Coulomb interaction $(p=2)$. In this case, one should trace out all irrelevant particles. In quantum chemistry, a particle reduction is realized by integrating over irrelevant particles' coordinates [28 30], which results in the so-called $p$-particle reduced density matrix (see, e.g., [31]). Such an approach provides new inequalities for fermionic occupation numbers and leads to the generalized Pauli constraints 32 35]. As far as systems with a varying number of particles are concerned, the construction of $p$-particle reduced density matrices is non-trivial and we discuss it in this paper. Moreover, we also consider the traced out part $\Phi^{p}(\varrho)$ with a varying number of particles. Thus, we generalize particle-reduction technique for systems with a varying number of quasiparticles and compare the spectra of $p$ particle reduced density matrix and $\Phi^{p}(\varrho)$.

As a by-product, we formalize the density matrix construction for states with a varying number of fermions. Our description of fermionic density operator differs from that used by Cahill and Glauber 36].

The problem of our interest - possible pure fermionic states with equispectral reduced density operators - is connected with the problem of calculating von Neumann entropy for the fermionic dynamical systems and estimating capacities of fermionic quantum channels [37, 38]. One may expect that the transfer of quantum information through a fermionic channel would be different from that for bosons [39], as the fermionic theory differs from the qubit theory [40, 41] in both tomography [42] and quantum computation [43].

The paper is organized as follows. In Sec. III we describe the notation used and formulate the problem. In Sec. III, the density matrix formalism is concisely clarified. In Sec. IV the main results regarding the spectra of mode-reduced states are obtained. In Sec. $\nabla]$ the construction and spectrum of particle-reduced operators are analyzed. In Sec. VI, brief conclusions are given.

\section{NOTATION}

Consider the algebra of canonical anticommutation relations (CAR algebra) $\mathcal{A}$ generated by the ladder operators $a_{s}, a_{s}^{\dagger}$ satisfying the relations [8, 44]

$$
\begin{aligned}
& a_{s} a_{t}^{\dagger}+a_{t}^{\dagger} a_{s}=\delta_{s t} I, \\
& a_{s} a_{t}+a_{t} a_{s}=a_{s}^{\dagger} a_{t}^{\dagger}+a_{t}^{\dagger} a_{s}^{\dagger}=0,
\end{aligned}
$$

where $s, t=1, \ldots, n$. Let $H_{n}$ be a Hilbert space with the dimension $\operatorname{dim} H_{n}=2^{n}$ and $\left\{\left|j_{1} \ldots j_{n}\right\rangle\right\}$ be a fixed orthonormal basis, where indices $j_{s}=0,1$. We shall suppose that $\mathcal{A}$ is realized as the algebra of operators in $H_{n}$ such that

$$
\begin{aligned}
& a_{t}^{\dagger}\left|j_{1} \ldots j_{n}\right\rangle \\
& =\left\{\begin{array}{l}
\exp \left(i \pi \sum_{s=1}^{t-1} j_{s}\right)\left|j_{1} \ldots j_{t-1} 1 j_{t+1} \ldots j_{n}\right\rangle \text { if } j_{t}=0 \\
0 \quad \text { if } \quad j_{t}=1
\end{array}\right.
\end{aligned}
$$

and $a_{t}$ is conjugate to $a_{t}^{\dagger}$.

A positive functional $\omega \in B\left(H_{n}\right)^{*}$ normed by the condition $\omega(I)=1$ is called a state on the algebra of all bounded operators $B\left(H_{n}\right)$ in $H_{n}$. Given a state $\omega$, there exists a unique positive unit-trace operator $\varrho$ such that $\omega(x)=\operatorname{Tr}(\varrho x), x \in B\left(H_{n}\right)$. A spectrum of $\omega$ is defined as the spectrum of $\varrho$. The following definition [45] will play a major role in our analysis:

Definition 1. The state $|\psi\rangle$ satisfies the parity superselection rule if a unit vector $|\psi\rangle \in H$ has the form

$$
|\psi\rangle=\sum_{j_{1}, \ldots, j_{n} \in\{0,1\}} \lambda_{j_{1} \ldots j_{n}}\left|j_{1} \ldots j_{n}\right\rangle, \lambda_{j_{1} \ldots j_{n}} \in \mathbb{C},
$$

where all the numbers $\sum_{s} j_{s}$ are even or odd alternatively for all non-zero $\lambda_{j_{1} \ldots j_{n}}$.

The set of all states satisfying the superselection rule contains the important subset consisting of pure states for which the number of particles $N$ is fixed. If this is the case, non-zero terms in (2) satisfy the condition $\sum_{s} j_{s}=$ $N=$ const. It is worth mentioning that all pure quasifree fermionic states have a fixed number of particles [46, 47].

Another important class of fermionic states is determined by their peculiar action on monomials of the odd order:

Definition 2. The state $\omega$ is said to be even if

$$
\omega\left(a_{s_{1}}^{\#} \ldots a_{s_{2 k+1}}^{\#}\right)=0
$$

for any choice of $2 k+1$ ladder operators $a_{s}^{\#}=a_{s}$ or $a_{s}^{\#}=a_{s}^{\dagger}$.

The relation between the above classes becomes especially clear in the case of pure states.

Proposition 1. The pure state $\omega$ is even iff it satisfies the parity superselection rule.

Proof. Suppose that $\omega$ satisfies the parity superselection rule. Consider the representation (2). Since the odd order monomial $a_{s_{1}}^{\#} \ldots a_{s_{2 k+1}}^{\#}$ changes even number of particles to the odd one and vice versa, the vectors 
$a_{s_{1}}^{\#} \ldots a_{s_{2 k+1}}^{\#}\left|j_{1} \ldots j_{n}\right\rangle$ and $\left|\tilde{j}_{1} \ldots \tilde{j}_{n}\right\rangle$ are orthogonal for any choice of $a_{s}^{\#}$ if $\left|j_{1} \ldots j_{n}\right\rangle$ and $\left|\tilde{j}_{1} \ldots \tilde{j}_{n}\right\rangle$ correspond to non-zero terms in Eq. (2). It follows that $\omega$ is even.

If $\omega$ does not satisfy the parity superselection rule, then its representation in the form of (2) contains at least two non-zero terms, say $\left|j_{1} \ldots j_{n}\right\rangle$ and $\left|\tilde{j}_{1} \ldots \tilde{j}_{n}\right\rangle$, such that $\sum_{s} j_{s}$ and $\sum_{s} \tilde{j}_{s}$ have different parity. There exists a unique monomial of the odd order $a_{s_{1}} \ldots a_{s_{2 k+1}}$, which is a partial isometrical operator mapping $\left|j_{1} \ldots j_{n}\right\rangle$ to $\left|\tilde{j}_{1} \ldots \tilde{j}_{n}\right\rangle$ and mapping all other basis vectors to zero. Thus, the value of $\omega$ is not equal to zero on this monomial. Hence, $\omega$ cannot be even.

Before we proceed to the analysis of mode and particle reductions of fermionic states, we construct the density matrix formalism in the convenient form.

\section{DENSITY MATRIX FOR FERMIONS}

Let us consider $n$ fermionic modes and the corresponding CAR algebra $\mathcal{A}, \operatorname{dim} \mathcal{A}=4^{n}$. The generators of $\mathcal{A}$ are $a_{s} a_{s}^{\dagger}, a_{s}, a_{s}^{\dagger}$, and $a_{s}^{\dagger} a_{s}, s=1, \ldots, n$. Binary representation of numbers $0, \ldots, 2^{n}-1$ forms the set of multiindices $\mathcal{J} \ni J=\left(j_{1}, \ldots, j_{n}\right), j_{s} \in\{0,1\}$. Given two multiindices $J, K \in \mathcal{J}$, let us define $A_{J K} \in \mathcal{A}$ by the formula

$$
A_{J K}=c_{j_{1}}^{\dagger} c_{j_{2}}^{\dagger} \ldots c_{j_{n}}^{\dagger} c_{k_{n}} \ldots c_{k_{2}} c_{k_{1}}
$$

where

$$
c_{j_{s}}^{\dagger}=\left\{\begin{array}{lll}
a_{s} a_{s}^{\dagger} & \text { if } & j_{s}=0, \\
a_{s}^{\dagger} & \text { if } & j_{s}=1 ;
\end{array} \quad c_{k_{s}}=\left\{\begin{array}{lll}
a_{s} a_{s}^{\dagger} & \text { if } & k_{s}=0, \\
a_{s} & \text { if } & k_{s}=1 .
\end{array}\right.\right.
$$

Proposition 2. The following relation holds:

$$
A_{J K}=\left|j_{1} \ldots j_{n}\right\rangle\left\langle k_{1} \ldots k_{n}\right| .
$$

Proof. Consider the operator $C_{K}=c_{k_{n}} \ldots c_{k_{1}}$, which is a rank one partial isometry in the sense that $C_{K}=|\varphi\rangle\langle\chi|$ for some unit vectors $|\varphi\rangle,|\chi\rangle \in H$ that are either orthogonal or coincide. Moreover, $C_{K}\left|k_{1} \ldots k_{n}\right\rangle=|0 \ldots 0\rangle$. Then, $A_{J K}=C_{J}^{\dagger} C_{K}$, which concludes the proof.

The construction similar to that in Eq. (3) was used in the paper [48] for other purposes.

Corollary 1. The element $\varrho \in \mathcal{A}$ defines a valid quantum state iff it can be represented in the form

$$
\varrho=\sum_{J, K \in \mathcal{J}} \lambda_{J K} A_{J K}
$$

where $\left(\lambda_{J K}\right)$ is a positive semidefinite matrix with the unit trace $\sum_{J} \lambda_{J J}=1$ (fermionic density matrix).

Proof. Any element of the algebra $\mathcal{A}$ can be represented in the form (5) because $\left(A_{J K}\right)$ are matrix units due to Proposition 2. The functional $\omega(x)=\sum_{J, K \in \mathcal{J}} \lambda_{J K} \operatorname{Tr}\left(A_{J K} x\right)$ gives non-negative values for positive semidefinite operators $x$ if and only if $\left(\lambda_{J K}\right)$ is a positive semidefinite matrix too (as the functional is a trace of the product of two matrices). Finally, $\omega(I)=\sum_{J} \lambda_{J J}=1$.
The $2^{n} \times 2^{n}$ matrix $\left(\lambda_{J K}\right)$ is a fermionic density matrix and fulfils the conventional requirements (Hermitian, positive semidefinite, and unit trace). The following Corollary provides a convenient description of the density operator.

Corollary 2. The $2^{n} \times 2^{n}$ matrix with elements $\left\langle A_{K J}\right\rangle$ is a density matrix of an $n$-mode fermionic state over which the average is taken.

Proof. In fact, $\left\langle A_{K J}\right\rangle=\operatorname{Tr}\left(A_{J K}^{\dagger} \varrho\right)=\lambda_{J K}$.

Example 1. Using Eq. (3) and Corollary 2, the 2-mode fermionic state can be described by the (total) density matrix

$$
\left(\begin{array}{cccc}
\left\langle a_{1} a_{1}^{\dagger} a_{2} a_{2}^{\dagger}\right\rangle & \left\langle a_{1} a_{1}^{\dagger} a_{2}^{\dagger}\right\rangle & \left\langle a_{1}^{\dagger} a_{2} a_{2}^{\dagger}\right\rangle & \left\langle a_{1}^{\dagger} a_{2}^{\dagger}\right\rangle \\
\left\langle a_{1} a_{1}^{\dagger} a_{2}\right\rangle & \left\langle a_{1} a_{1}^{\dagger} a_{2}^{\dagger} a_{2}\right\rangle & \left\langle a_{1}^{\dagger} a_{2}\right\rangle & \left\langle a_{1}^{\dagger} a_{2}^{\dagger} a_{2}\right\rangle \\
\left\langle a_{1} a_{2} a_{2}^{\dagger}\right\rangle & \left\langle a_{2}^{\dagger} a_{1}\right\rangle & \left\langle a_{1}^{\dagger} a_{1} a_{2} a_{2}^{\dagger}\right\rangle & \left\langle a_{1}^{\dagger} a_{2}^{\dagger} a_{1}\right\rangle \\
\left\langle a_{2} a_{1}\right\rangle & \left\langle a_{1} a_{2}^{\dagger} a_{2}\right\rangle & \left\langle a_{1}^{\dagger} a_{2} a_{1}\right\rangle & \left\langle a_{1}^{\dagger} a_{1} a_{2}^{\dagger} a_{2}\right\rangle
\end{array}\right),
$$

which is constructed with the help of conventional sets of multiindices $\mathcal{J}, \mathcal{K}=\{(0,0) ;(0,1) ;(1,0) ;(1,1)\}$ and differs from symbols used in the description of fermionic Gaussian states [49] (reviewed, e.g., in [50]).

\section{SPECTRA OF MODE-REDUCED STATES}

In this section, we deal with coherent superpositions of different number states, so we exploit the bipartition based not on the particles but rather on fermionic modes. Fermionic modes can be thought of as nodes that can be either occupied or not by particles. The bipartition is merely an aggregation of all nodes into two groups. For instance, bipartitions with respect to different spin components were considered recently [51]. Mathematically, we deal with the algebraic bipartition in the second quantized description 52 56], which is used in the study of entanglement [45, 57,60].

Partition of modes is performed among single-particle states that fermions can potentially occupy, for example, nodes of some lattice potential in coordinate configuration space, or states with different momentum in momentum configuration space. Let the first subsystem contain $m$ modes and the second one contain $n-m$ modes. In mathematical terms, $\mathcal{A}$ is obtained by joining two algebras of canonical anticommutation relations $\mathcal{A}_{1}$ and $\mathcal{A}_{2}$ with the generators $\left\{a_{1}, a_{1}^{\dagger}, \ldots, a_{m}, a_{m}^{\dagger}\right\}$ and $\left\{a_{m+1}, a_{m+1}^{\dagger}, \ldots, a_{n}, a_{n}^{\dagger}\right\}$, respectively. Fix a unit vector $|\psi\rangle \in H$.

Definition 3. The states $\omega_{j}(x)=\langle\psi|x| \psi\rangle, x \in \mathcal{A}_{j}$ that are the restrictions of a pure state $|\psi\rangle\langle\psi|$ to the algebras $\mathcal{A}_{j}, j=1,2$, are said to be mode-reduced (partial) states of $|\psi\rangle\langle\psi|$.

Example 2. For a state (6) the reduced density matrices describing the first mode and the second mode read

$$
\left(\begin{array}{cc}
\left\langle a_{1} a_{1}^{\dagger}\right\rangle & \left\langle a_{1}^{\dagger}\right\rangle \\
\left\langle a_{1}\right\rangle & \left\langle a_{1}^{\dagger} a_{1}\right\rangle
\end{array}\right) \text { and }\left(\begin{array}{cc}
\left\langle a_{2} a_{2}^{\dagger}\right\rangle & \left\langle a_{2}^{\dagger}\right\rangle \\
\left\langle a_{2}\right\rangle & \left\langle a_{2}^{\dagger} a_{2}\right\rangle
\end{array}\right)
$$

respectively. Note that both matrices (7) cannot be simultaneously obtained from the total density matrix (6) 
by conventional partial trace methods. This is a peculiar property of fermionic states which differs them from the bosonic ones.

Remark 1. Let us clarify the relation between the introduced definition of the mode-reduced states and the orbital reduced density matrices used in quantum chemistry 61, 62.

In quantum chemistry, the mode is a composite of the space orbital state and the electron spin projection $(|\uparrow\rangle$ or $|\downarrow\rangle)$, and the whole system state vector is written in the form $|\Psi\rangle=\sum_{n_{1}, \ldots, n_{L}} \psi_{n_{1}, \ldots, n_{L}}\left|n_{1}\right\rangle \otimes \ldots \otimes\left|n_{L}\right\rangle$, where $n_{i}=0,1$ indicates the occupation of the corresponding spin-orbit, $L$ is the number of active spin-orbits. The antisymmetric property of the electron wavefunction is encoded in the coefficients $\psi_{n_{1}, \ldots, n_{L}}$, and the global basis states $\left|n_{1}\right\rangle \otimes \ldots \otimes\left|n_{L}\right\rangle$ already have the tensor product structure. The reduced states are then readily obtained by tracing out undesired orbits.

Alternatively, one can use antisymmetric vectors (Slater determinants) $\left|e_{1}\right\rangle=\left|n_{1} \ldots n_{i-1}\right\rangle, \quad\left|e_{2}\right\rangle=$ $\left|n_{i+1} \ldots n_{j-1}\right\rangle,\left|e_{3}\right\rangle=\left|n_{j+1} \ldots n_{L}\right\rangle$ and expand $|\Psi\rangle=$ $\sum_{n_{i}, n_{j}, e_{1}, e_{2}, e_{3}} \psi_{n_{i}, n_{j}, e_{1}, e_{2}, e_{3}}\left|e_{1}\right\rangle \otimes\left|n_{i}\right\rangle \otimes\left|e_{2}\right\rangle \otimes\left|n_{j}\right\rangle \otimes\left|e_{3}\right\rangle$. The reduced 2-mode state reads $\operatorname{Tr}_{e_{1}, e_{2}, e_{3}}|\Psi\rangle\langle\Psi|$ as each vector $\left|e_{1}\right\rangle \otimes\left|n_{i}\right\rangle \otimes\left|e_{2}\right\rangle \otimes\left|n_{j}\right\rangle \otimes\left|e_{3}\right\rangle$ does have a tensor product structure. Using the orbit basis $\left|n_{i}\right\rangle=\{|0\rangle, \mid \uparrow$ \rangle$,|\downarrow\rangle,|\uparrow \downarrow\rangle\}$, one gets the 2-orbital reduced density operator 61].

In our approach, the global basis states $\left|j_{1} \ldots j_{n}\right\rangle$ are antisymmetric automatically and coefficients $\lambda_{j_{1} \ldots j_{n}}$ in Eq. (2) are arbitrary. Thus, we deal with basis states that do not have the tensor product structure at all. Similarly, the action of mode operators $a_{t}$ and $a_{t}^{\dagger}$ is nonlocal. Since transitions between the states with different numbers of particles are prohibited in quantum chemistry 61, 62], both quantum-chemical and our definitions of reduced states do coincide for systems with a fixed number of electrons. The downside of the quantum-chemical definition may only appear for systems with variable number of particles, since the creation and annihilation operators cannot be represented in the tensor product form $I \otimes \ldots \otimes I \otimes|1\rangle\langle 0| \otimes I \otimes \ldots \otimes I$ or $I \otimes \ldots \otimes I \otimes|0\rangle\langle 1| \otimes I \otimes \ldots \otimes I$.

Our further goal is to characterize all the states that have equispectral mode-reduced states. To anticipate general results, let us begin with the simplest case of two fermionic modes that can be occupied by a system with a varying number of quasiparticles in a pure state $|\psi\rangle=c_{00}|00\rangle+c_{01}|01\rangle+c_{10}|10\rangle+c_{11}|11\rangle$. According to Example1 the total density matrix (6) for such a state is $\left(c_{00}, c_{01}, c_{10}, c_{11}\right)^{\top} \times\left(\overline{c_{00}}, \overline{c_{01}}, \overline{c_{10}}, \overline{c_{11}}\right)$ and corresponds to the pure state indeed, whereas the reduced density matrices (7) take the form

$$
\begin{aligned}
& \Lambda_{1}=\left(\begin{array}{cc}
\left|c_{00}\right|^{2}+\left|c_{01}\right|^{2} & c_{00} \overline{c_{10}}+c_{01} \overline{c_{11}} \\
\overline{c_{00}} c_{10}+\overline{c_{01}} c_{11} & \left|c_{10}\right|^{2}+\left|c_{11}\right|^{2}
\end{array}\right) \text { and } \\
& \Lambda_{2}=\left(\begin{array}{cc}
\left|c_{00}\right|^{2}+\left|c_{10}\right|^{2} & c_{00} \overline{c_{01}}-c_{10} \overline{c_{11}} \\
\overline{c_{00}} c_{01}-\overline{c_{10}} c_{11} & \left|c_{01}\right|^{2}+\left|c_{11}\right|^{2}
\end{array}\right),
\end{aligned}
$$

respectively. The spectra of $\Lambda_{1}$ and $\Lambda_{2}$ would be identical if and only if $\operatorname{Tr}\left(\Lambda_{1}\right)=\operatorname{Tr}\left(\Lambda_{2}\right)$ and $\operatorname{Tr}\left(\Lambda_{1}^{2}\right)=\operatorname{Tr}\left(\Lambda_{2}^{2}\right)$. The first condition always holds true whereas the second one reduces to $\operatorname{Tr}\left(\Lambda_{1}^{2}-\Lambda_{2}^{2}\right)=8 \operatorname{Re}\left(c_{00} c_{11} \overline{c_{01} c_{10}}\right)=0$. This observation can be summarized as follows.

Proposition 3. The two-mode fermionic state $|\psi\rangle=$ $c_{00}|00\rangle+c_{01}|01\rangle+c_{10}|10\rangle+c_{11}|11\rangle$ has equispectral modereduced density operators if and only if $\operatorname{Re}\left(c_{00} c_{11} \overline{c_{01} c_{10}}\right)=$ 0 .

Example 3. Suppose $|\psi\rangle=\frac{1}{2}(|00\rangle+|01\rangle+|10\rangle+|11\rangle)$, then $\operatorname{Spect}\left(\omega_{1}\right)=\{0,1\}$ whereas $\operatorname{Spect}\left(\omega_{2}\right)=\left\{\frac{1}{2}, \frac{1}{2}\right\}$.

Theorem 1. Suppose that a pure state $\omega$ satisfies the parity superselection rule. Then, the spectra of $\omega_{1}$ and $\omega_{2}$ coincide.

Proof. Let us define an isometry $U: H_{n} \rightarrow H_{m} \otimes H_{n-m}$ by the formula

$$
U\left|j_{1} \ldots j_{n}\right\rangle=\left|j_{1} \ldots j_{m}\right\rangle \otimes\left|j_{m+1} \ldots j_{n}\right\rangle .
$$

Then

$$
U a_{s}^{\#} U^{\dagger}=\left\{\begin{array}{lll}
a_{s}^{(1) \#} \otimes I & \text { if } & s=1, \ldots, m \\
\Gamma \otimes a_{s}^{(2) \#} & \text { if } & s=m+1, \ldots, n
\end{array}\right.
$$

where the fermionic operators $a_{s}^{(1) \#}$ and $a_{s}^{(2) \#}$ act on Hilbert spaces $H_{m}$ and $H_{n-m}$, respectively, and $\Gamma=$ $\prod_{s=1}^{m}\left(a_{s}^{(1)} a_{s}^{(1) \dagger}-a_{s}^{(1) \dagger} a_{s}^{(1)}\right)$.

Consider the pure state $\Omega(X)=\omega\left(U^{\dagger} X U\right), X \in$ $B\left(H_{m} \otimes H_{n-m}\right)$. The spectra of $\Omega_{1}=\operatorname{Tr}_{H_{n-m}}(\Omega)$ and $\Omega_{2}=\operatorname{Tr}_{H_{m}}(\Omega)$ are known to coincide as an immediate consequence of the Schmidt decomposition in tensor product Hilbert space $H_{m} \otimes H_{n-m}$ (see, e.g., 63] ). It follows from the construction that for all $x \in \mathcal{A}_{1}$ we have

$$
\omega_{1}(x)=\omega(x)=\Omega\left(U x U^{\dagger}\right)=\Omega\left(x^{(1)} \otimes I\right)=\Omega_{1}(x),
$$

therefore, $\omega_{1}$ and $\Omega_{1}$ have the same spectra.

To prove that spectra of $\omega_{2}$ and $\Omega_{2}$ coincide too, we first notice the trivial action of these functionals on odd order monomials, namely, $\omega_{2}\left(a_{s_{1}}^{\#} \ldots a_{s_{2 k+1}}^{\#}\right)=$ $\omega\left(a_{s_{1}}^{\#} \ldots a_{s_{2 k+1}}^{\#}\right)=0$ because $\omega$ is even state in virtue of Proposition [1] On the other hand, for all $m+1 \leq s_{p} \leq n$

$$
\begin{aligned}
& \Omega_{2}\left(a_{s_{1}}^{(2) \#} \ldots a_{s_{2 k+1}}^{(2) \#}\right)=\Omega\left(I \otimes a_{s_{1}}^{(2) \#} \ldots a_{s_{2 k+1}}^{(2) \#}\right) \\
& =\omega\left(\prod_{s=1}^{m}\left(a_{s} a_{s}^{\dagger}-a_{s}^{\dagger} a_{s}\right) a_{s_{1}}^{\#} \ldots a_{s_{2 k+1}}^{\#}\right)=0
\end{aligned}
$$

because $\omega$ is even. Thus, both $\omega_{2}$ and $\Omega_{2}$ vanish on odd monomials. As far as even monomials are concerned,

$$
\begin{aligned}
& \omega\left(a_{s_{1}}^{\#} \ldots a_{s_{2 k}}^{\#}\right)=\Omega\left(U a_{s_{1}}^{\#} \ldots a_{s_{2 k}}^{\#} U^{\dagger}\right) \\
& =\Omega\left(\Gamma^{2 k} \otimes a_{s_{1}}^{(2) \#} \ldots a_{s_{2 k}}^{(2) \#}\right)=\Omega_{2}\left(s_{s_{1}}^{(2) \#} \ldots a_{s_{2 k}}^{(2) \#}\right)
\end{aligned}
$$

because $\Gamma^{2 k}=I$. Thus, $\omega_{2}$ and $\Omega_{2}$ coincide on even monomials too. To conclude, $\operatorname{Spect}\left(\omega_{1}\right)=\operatorname{Spect}\left(\Omega_{1}\right)=$ $\operatorname{Spect}\left(\Omega_{2}\right)=\operatorname{Spect}\left(\omega_{2}\right)$.

The obtained result shows that states satisfying the parity superselection rule have equispectral mode-reduced states and, therefore, are physical. Note that superselected states do not necessary have a fixed number of particles, and we believe that a coherent superposition (not a mixture) of states with different numbers of fermionic 
quasiparticles can be observed in future experiments 20 22]. From a mathematical viewpoint, it is interesting to find all possible states with equispectral mode-reduced states. The answer to this question provides the following theorem.

Theorem 2. Suppose that for a pure state $|\psi\rangle\langle\psi|$ the partial states $\omega_{1}$ and $\omega_{2}$ have identical spectra. Then, there exist a state $|\phi\rangle\langle\phi|$ satisfying the parity superselection rule and unitary operators $U_{1} \in \mathcal{A}_{1}, U_{2} \in \mathcal{A}_{2}$ such that the partial states of $\left|U_{2} U_{1} \phi\right\rangle\left\langle U_{2} U_{1} \phi\right|$ coincide with $\omega_{i}, i=1,2$. If the spectra of $\omega_{i}$ are simple (nondegenerate), then $|\psi\rangle=U_{1} U_{2}|\phi\rangle$.

Proof. Without loss of generality it can be assumed that bipartition of $n$ fermionic modes into $m$ and $n-m$ modes is such that $n-m \leq m$ (otherwise the modes can be relabelled).

Consider the state $\omega_{2}$. In the Hilbert space $H_{n-m}$ choose the orthonormal basis $\left\{\left|e_{j_{m+1} \ldots j_{n}}\right\rangle\right\}$ such that for all $x \in \mathcal{A}_{2}$

$$
\omega_{2}(x)=\sum_{j_{s}=0,1: m+1 \leq s \leq n} \lambda_{j_{m+1} \ldots j_{n}}\left\langle e_{j_{m+1} \ldots j_{n}}|x| e_{j_{m+1} \ldots j_{n}}\right\rangle .
$$

Pick the unitary operator $U_{2} \in \mathcal{A}_{2}$ such that

$U_{2}\left|j_{m+1} \ldots j_{n}\right\rangle=\left|e_{j_{m+1} \ldots j_{n}}\right\rangle, \quad j_{s} \in\{0,1\}, \quad m+1 \leq s \leq n$.

Consider the pure state $\tilde{\omega}(x)=\omega\left(U_{2} x U_{2}^{\dagger}\right), x \in \mathcal{A}$. Then for all $x \in \mathcal{A}_{2}$ we have

$\tilde{\omega}_{2}(x)=\sum_{j_{s}=0,1: m+1 \leq s \leq n} \lambda_{j_{m+1} \ldots j_{n}}\left\langle j_{m+1} \ldots j_{n}|x| j_{m+1} \ldots j_{n}\right\rangle$

Since $\omega_{1}$ and $\omega_{2}$ are equispectral by the statement of Theorem, the states $\tilde{\omega}_{1}$ and $\tilde{\omega}_{2}$ are equispectral too due to the local action of $U_{2}$. Consequently, there exists the orthonormal basis $\left\{\left|f_{t}\right\rangle\right\}_{t=0}^{2^{n-m}-1} \cup\left\{\left|g_{t}\right\rangle\right\}_{t=2^{n-m}}^{2^{m}-1}$ in $H_{m}$ such that for all $x \in \mathcal{A}_{1}$

$$
\tilde{\omega}_{1}(x)=\sum_{t=0}^{2^{n-m}-1} \lambda_{j_{m+1} \ldots j_{n}=\operatorname{bin}(t)}\left\langle f_{t}|x| f_{t}\right\rangle,
$$

where $\operatorname{bin}(t)$ is the binary representation of $t$. Pick the unitary operator $U_{1} \in \mathcal{A}_{1}$ such that

$$
\begin{gathered}
U_{1}|\underbrace{0 \ldots 0}_{\begin{array}{c}
2 m-n \\
\text { bits }
\end{array}} \underbrace{\operatorname{bin}(t)}_{\begin{array}{c}
n-m \\
\text { bits }
\end{array}}\rangle=\left|f_{t}\right\rangle, \quad t=0, \ldots, 2^{n-m}-1, \\
U_{1}|\underbrace{\operatorname{bin}(t)}_{\begin{array}{c}
m \\
\text { bits }
\end{array}}\rangle=\left|g_{t}\right\rangle, \quad t=2^{n-m}, \ldots, 2^{m}-1 .
\end{gathered}
$$

It is not hard to see that a vector

$$
|\phi\rangle=\sum_{t=0}^{2^{n-m}} \sqrt{\lambda_{j_{m+1} \ldots j_{n}=\operatorname{bin}(t)}}|\underbrace{0 \ldots 0}_{\begin{array}{c}
2 m-n \\
\text { bits }
\end{array}} \underbrace{\operatorname{bin}(t)}_{\begin{array}{c}
n-m \\
\text { bits }
\end{array}} \underbrace{\operatorname{bin}(t)}_{\begin{array}{c}
n-m \\
\text { bits }
\end{array}}\rangle
$$

generates the state $\Omega(x)=\langle\phi|x| \phi\rangle, x \in \mathcal{A}$, which satisfies the parity superselection rule as the number of quasiparticles in $t$-th summand equals an even number $2 \times(\#$ of 1 's in $\operatorname{bin}(\mathrm{t}))$. Moreover, $\Omega$ has $\tilde{\omega}_{2}$ and $\tilde{\tilde{\omega}}_{1}(x)=$ $\tilde{\omega}_{1}\left(U_{1} x U_{1}^{\dagger}\right)$ as its partial states. In other words, the modereduced states of $|\psi\rangle\langle\psi|$ and the mode-reduced states of $\left|U_{2} U_{1} \phi\right\rangle\left\langle U_{2} U_{1} \phi\right|$ coincide.

Now suppose that the coincident spectra of $\omega_{i}, i=1,2$ are simple (nondegenerate). Take the unitary operators $U$ and $\Gamma$ determined in Eq. (8) and consider the state $\tilde{\Omega}(X)=\Omega\left(U^{\dagger} X U\right), X \in \mathcal{A}_{1} \otimes \mathcal{A}_{2}$. According to Eq. (10), for all $x \in \mathcal{A}_{1}$ we have $\tilde{\Omega}_{1}(x)=\tilde{\tilde{\omega}}_{1}(x)$. Analogously, since $\Omega$ is even, from Eqs. (11) $-(12)$ it follows that for all $x \in \mathcal{A}_{2}$ the relation $\tilde{\Omega}_{2}(x)=\tilde{\omega}_{2}(x)$ holds.

On the other hand, as the spectrum of $\Omega_{i}$ is simple there exists the only state on $\mathcal{A}_{1} \otimes \mathcal{A}_{2}$ (namely, $\tilde{\Omega}$ ) such that its partial traces coincide with $\Omega_{1}$ and $\Omega_{2}$ (see, e.g., 63]). Hence, the same uniqueness holds for the state on $\mathcal{A}$ (namely, $\Omega$ ). Thus, there is the only possibility to reconstruct the entire state from its partial states. It implies that $\omega(x)=\Omega\left(U_{1}^{\dagger} U_{2}^{\dagger} x U_{2} U_{1}\right)$ for all $x \in \mathcal{A}$.

Theorem 2 characterizes possible states with equispectral mode-reduced states. Such states do not have to satisfy the parity superselection rule (cf. Proposition 3) but they are obtained from the superselected states by unitary operators acting on corresponding parties of the bipartite state.

\section{SPECTRA OF PARTICLE-REDUCED OPERATORS}

In this section, we consider reductions over particles. When the number of particles $N$ is fixed, as it takes place in quantum chemistry, the reduction is performed by integrating the density operator $\varrho\left(x_{1}, \ldots, x_{N}, x_{1}^{\prime}, \ldots, x_{N}^{\prime}\right)$ over some particles' coordinates [28 31]. This results in the so-called $p$-particle reduced density matrix ( $p$-RDM):

$$
\begin{aligned}
& \varrho_{p-\mathrm{RDM}}\left(x_{1}, \ldots, x_{p} ; x_{1}^{\prime}, \ldots, x_{p}^{\prime}\right) \\
& =\int \varrho\left(x_{1}, \ldots, x_{p}, x_{p+1}, \ldots, x_{N} ; x_{1}^{\prime}, \ldots, x_{p}^{\prime}, x_{p+1}, \ldots, x_{N}\right) \\
& \times d x_{p+1} \ldots d x_{N} \cdot(15)
\end{aligned}
$$

If a pure fermionic state $\varrho$ has exactly $N$ particles, then the spectra of reduced density matrices $\varrho_{p \text {-RDM }}$ and $\varrho_{(N-p) \text {-RDM }}$ are known to coincide [28]. Eigenvalues of 1 -RDM are called natural occupation numbers. In general case, natural occupation numbers cannot be arbitrary ones within $[0,1]$ (simple Pauli's exclusion principle) and must satisfy generalized Pauli constraints 32 35].

Let us generalize the construction of reduced density matrices for states with a varying number of particles. In the second quantization formalism, the integration in Eq. (15) takes the form

$$
\varrho_{p-\mathrm{RDM}}\left(s_{1}, \ldots, s_{p} ; t_{1}, \ldots, t_{p}\right)=\left\langle a_{s_{1}}^{\dagger} \ldots a_{s_{p}}^{\dagger} a_{t_{p}} \ldots a_{t_{1}}\right\rangle
$$

where $s_{q}, t_{q}=1, \ldots, n$, i.e. all modes are accessible. Note that all $s_{q}$ are to be different and all $t_{q}$ are to be different for expression (16) not to vanish.

The definition (16) implies that $p$-RDM does not have unit trace (in contrast to Eq. (15)). If the number of particles in the state $\varrho$ is fixed and equals $N$, then one 
can introduce a factor $\frac{(N-p) !}{N !}$ in the right-hand side of Eq. (16) to make its trace unit 29]. As our aim is to deal with general states that do not have a fixed number of particles, we do not introduce any factors in the righthand side of Eq. (16).

As far as the states $\varrho$ with a variable number of particles are concerned, average values (16) are still meaningful and can be calculated. This enables one to construct the particle-reduced density operator in $B\left(H_{n}\right)$ as follows:

$$
\begin{gathered}
\varrho_{p}=\sum_{\substack{s_{1}<\ldots<s_{p} \\
t_{1}<\ldots<t_{p}}}\left\langle a_{s_{1}}^{\dagger} \ldots a_{s_{p}}^{\dagger} a_{t_{p}} \ldots a_{t_{1}}\right\rangle a_{t_{1}}^{\dagger} \ldots a_{t_{p}}^{\dagger} a_{s_{p}} \ldots a_{s_{1}} \\
\times \prod_{\substack{s \neq s_{1}, \ldots, s_{p}, t_{1}, \ldots, t_{p}}} a_{s} a_{s}^{\dagger} .
\end{gathered}
$$

Note that the number of particles in the operator (17) is fixed without regard to a possible variable number of particles in the original state. Product $\prod_{s}$ in formula (17) is responsible for unoccupied modes.

Example 4. The two-mode fermionic state $|\psi\rangle=$ $c_{00}|00\rangle+c_{01}|01\rangle+c_{10}|10\rangle+c_{11}|11\rangle$ has 1-RDM of the form $\left(\begin{array}{cc}\left|c_{10}\right|^{2}+\left|c_{11}\right|^{2} & c_{01} \overline{c_{10}} \\ \overline{c_{01}} c_{10} & \left|c_{01}\right|^{2}+\left|c_{11}\right|^{2}\end{array}\right)$ whose spectrum is $\left(\left|c_{01}\right|^{2}+\left|c_{10}\right|^{2}+\left|c_{11}\right|^{2},\left|c_{11}\right|^{2}\right)=\left(1-\left|c_{00}\right|^{2},\left|c_{11}\right|^{2}\right)$. There are no constraints on natural occupation numbers $\lambda_{1}, \lambda_{2} \in$ $[0,1]$. This is in agreement with a general statement that in Fock space there are no constraints on the spectra of the 1-RDM other than those imposed by the Pauli's exclusion principle [64]. However, if we restrict $|\psi\rangle$ to satisfy the parity superselection rule, then the generalized constraint on natural occupation numbers appears: $\lambda_{1}=\lambda_{2}$ for states with even parity, $\lambda_{2}=0$ for states with odd parity.

Example 5. Odd parity 3-mode fermionic state $c_{100}|100\rangle+c_{010}|010\rangle+c_{001}|001\rangle+c_{111}|111\rangle$ has natural occupation numbers $\lambda_{1}=\left|c_{100}\right|^{2}+\left|c_{010}\right|^{2}+\left|c_{001}\right|^{2}+\left|c_{111}\right|^{2}=$ $1, \lambda_{2}=\lambda_{3}=\left|c_{111}\right|^{2}$. The requirements $\lambda_{1}=1$ and $\lambda_{2}=\lambda_{3}$ may be considered as generalized Pauli constraints in this case.

Suppose the physical process of tracing out 1 particle from a general fermionic state with a variable number of particles. The result of this operation still contains a variable number of particles so it cannot be described by a reduced density matrix (16). This operation is rather described by the map

$$
\Phi(\varrho)=\sum_{j} a_{j} \varrho a_{j}^{\dagger} .
$$

If the state $\varrho$ has a fixed number of particles, say $N$, then $\Phi(\varrho)$ is an $N-1$ particle operator, which coincides with the reduced density operator $\varrho_{N-1}$ given by formula (17) (see the proof below). Thus, the map $\Phi$ can be considered as a generalization of integration over one particle coordinates. Sequentially applying this map $p$ times we get $\Phi^{p}$, which is nothing else but tracing out $p$ particles.

Proposition 4. Suppose the density operator @ has a fixed number $N$ of particles, then $\Phi^{N-p}(\varrho)=\varrho_{p}$.
Proof. According to Corollary $11, \varrho=$ $\sum_{|J|=|K|=N} \lambda_{J K} A_{J K}$, where $|J|=\sum_{s} j_{s}$ is the number of particles present in the multiindex $J$. Using the explicit form of operators (4), we get

$$
\begin{gathered}
A_{J K}=\underbrace{a_{s_{1}}^{\dagger} \ldots a_{s_{N}}^{\dagger} a_{t_{N}} \ldots a_{t_{1}}}_{s_{1}<\ldots<s_{N}: j_{s_{q}}=1} \prod_{s: j_{s}=k_{s}=0} a_{s} a_{s}^{\dagger}, \\
a_{l} A_{J K} a_{l}^{\dagger}=\left\{\begin{array}{l}
a_{s_{1}}^{\dagger} \ldots a_{l} \ldots a_{s_{N}}^{\dagger} a_{t_{N}} \ldots a_{l}^{\dagger} \ldots a_{t_{1}} \\
\times \prod_{s: j_{s}=k_{s}=0} a_{s} a_{s}^{\dagger} \text { if } j_{l}=k_{l}=1, \\
0 \text { otherwise, }
\end{array}\right.
\end{gathered}
$$

which follows from $a_{l} a_{l}^{\dagger} a_{l} a_{l}^{\dagger}=a_{l} a_{l}^{\dagger}$. On the other hand, substituting $A_{J K}$ for $\varrho$ in formula (17) yields

$$
\begin{gathered}
\left(A_{J K}\right)_{N-1}=\sum_{l: j_{l}=k_{l}=1} a_{s_{1}}^{\dagger} \ldots a_{l} \ldots a_{s_{N}}^{\dagger} a_{t_{N}} \ldots a_{l}^{\dagger} \ldots a_{t_{1}} \\
\times \prod_{s: j_{s}=k_{s}=0} a_{s} a_{s}^{\dagger}, \quad(21)
\end{gathered}
$$

which implies that $\Phi(\varrho)=\varrho_{N-1}$. Applying this relation $N-p$ times, we get the statement of Proposition 4 .

Corollary 3. The operators $\varrho_{p}$ and $\Phi^{p}(\varrho)$ have the same spectra for pure states $\varrho$ with a fixed number of particles.

Proof. The statement is an immediate consequence of Proposition 4 and the fact that for pure states $\operatorname{Spec}\left(\varrho_{p}\right)=$ $\operatorname{Spec}\left(\varrho_{N-p}\right)[28]$.

Example 6. The spectra of $\varrho_{p}$ and $\Phi^{p}(\varrho)$ may coincide not only for states with a fixed number of particles. The coincidence of spectra of $\varrho_{1}$ and $\Phi(\varrho)$ takes place for a general two-mode fermionic state $|\psi\rangle=c_{00}|00\rangle+c_{01}|01\rangle+$ $c_{10}|10\rangle+c_{11}|11\rangle$. It is not hard to see from the matrix representation (6) of $\Phi(|\psi\rangle\langle\psi|)$ which reads

$$
\left(\begin{array}{cccc}
\left|c_{01}\right|^{2}+\left|c_{10}\right|^{2} & c_{10} \overline{c_{11}} & -c_{01} \overline{c_{11}} & 0 \\
\overline{c_{10}} c_{11} & \left|c_{11}\right|^{2} & 0 & 0 \\
-\overline{c_{01}} c_{11} & 0 & \left|c_{11}\right|^{2} & 0 \\
0 & 0 & 0 & 0
\end{array}\right)
$$

Eigenvalues of (22) are the same as those of 1-RDM found in Example 4.

Consideration of other examples (general 3- and 4-mode pure states) also results in equispectral particle reductions $\varrho_{p}$ and $\Phi^{p}(\varrho)$. We can make a conjecture that spectra of operators $\varrho_{p}$ and $\Phi^{p}(\varrho)$ coincide for any pure fermionic state $\varrho$.

\section{CONCLUSIONS}

We have constructed mode- and particle-reduced fermionic density operators for states with a varying number of particles and analyzed their spectra. The mode reduction is based on the restriction functional $\omega(x)=$ $\operatorname{Tr}(\varrho x)$ to subalgebra, whereas the particle reduction is realized via two objects: $p$-particle operator $\varrho_{p}$ and the result of tracing out $p$ particles, $\Phi^{p}(\varrho)$. As a byproduct, we have analyzed the density matrix formalism for general case of fermionic states and provided the explicit 
construction of density matrices (Corollary 2 and Example1). The developed formalism clearly indicates that the conventional partial trace methods are not applicable to fermionic states.

We have addressed the problem of finding general pure fermionic states such that their mode-reduced density matrices are equispectral. Equispectrality automatically takes place for bosonic systems and systems of distinguishable particles, however, it does not necessarily hold true for systems composed of indistinguishable fermionic quasiparticles. On the other hand, equispectrality is a natural quantum information property that reflects the fact that entropies of subsystems must coincide.

For mode-reduced states, we have found necessary and sufficient conditions for their spectra to be identical. The results of Proposition 3 and Theorem 2 can also be interpreted as the construction of valid purifications for fermionic density operators. Purification is a frequently used tool in quantum information theory, and we believe that these results may turn out to be useful in characterization of fermionic channels.

For particle-reduced states, we have shown that $\Phi^{p}(\varrho)$ is a valid reduction, which coincides with $\varrho_{N-p}$ if the state $\varrho$ has exactly $N$ particles. Basing on the examples, we have conjectured that spectra of $\varrho_{p}$ and $\Phi^{p}(\varrho)$ coincide not only for $N$-particle states but also for a general fermionic pure state $\varrho$. We have demonstrated that the natural occupation numbers $\lambda_{1} \geq \ldots \geq \lambda_{n}$ (spectrum of 1 -RDM) must obey generalized Pauli constraints not only for $N$-particle states but also for states satisfying the parity superse- lection rule. For instance, even parity two-mode states necessarily satisfy $\lambda_{1}=\lambda_{2}$, odd parity two-mode states have $\lambda_{2}=0$, and odd parity three-mode states fulfil the requirements $\lambda_{1}=1, \lambda_{2}=\lambda_{3}$. The generalization of these constraints is a problem for a future research.

\section{Acknowledgments}

The authors are delighted to thank A.S. Holevo for a motivation of this work and illuminating discussions. The authors are grateful to Christian Schilling for fruitful discussions and bringing Ref. 64] to our attention. Propositions 1 and 2, Corollary 1, Theorems 1 and 2 are proved by G.G. Amosov. Corollary 2. Proposition 3, Examples 1 and 3 . Section $\mathrm{V}$ ] are due to S.N. Filippov. Both authors discussed the results and commented on the manuscript. The work of G.G. Amosov is supported by Russian Science Foundation under grant No. 14-21-00162 and performed in Steklov Mathematical Institute of Russian Academy of Sciences. S.N. Filippov's work on Sections 3 and 4 is supported by the Russian Foundation for Basic Research under Project No. 16-37-60070 mol_a_dk and performed in Institute of Physics and Technology, Russian Academy of Sciences. S.N. Filippov's work on Sections 5 is supported by Russian Science Foundation under project No. 16-1100084 and performed in Moscow Institute of Physics and Technology.
[1] Landauer, R.: The physical nature of information. Phys. Lett. A 217, 188 (1996)

[2] Nielsen, M.A., Chuang, I.L.: Quantum Computation and Quantum Information. Cambridge University Press, Cambridge (2000)

[3] Pauli, W.: Über den Einflußder Geschwindigkeitsabhängigkeit der Elektronenmasse auf den Zeemaneffekt. Zeitschrift für Physik 31, 373 (1925)

[4] Uhlenbeck, G.E., Goudsmit, S.: Zuschriften und vorläufige Mitteilungen. Die Naturwissenschaften 13, 953 (1925)

[5] Pauli, W.: Über den Zusammenhang des Abschlusses der Elektronengruppen im Atom mit der Komplexstruktur der Spektren. Zeitschrift für Physik 31, 765 (1925)

[6] Fermi, E.: Zur Quantelung des idealen einatomigen Gases. Zeitschrift für Physik 36, 902 (1926)

[7] Dirac, P.A.M.: On the theory of quantum mechanics. Proc. Royal Soc. A 112, 661 (1926)

[8] Jordan, P., Wigner, E.P.: Über das Paulische Äquivalenzverbot. Zeitschrift für Physik 47, 631 (1928)

[9] Fock, V.: Konfigurationsraum und zweite Quantelung. Zeitschrift für Physik 75, 622 (1932)

[10] Fierz, M.: Über die relativistische Theorie kräftefreier Teilchen mit beliebigem Spin. Helvetica Physica Acta 12, 3 (1939)

[11] Pauli, W.: The connection between spin and statistics. Phys. Rev. 58, 716 (1940)

[12] Wick, G.C., Wightman, A.S., Wigner, E.P.: The intrinsic parity of elementary particles. Phys. Rev. 88, 101 (1952)

[13] Wightman, A.S.: Superselection rules; Old and new. Il
Nuovo Cimento B 110, 751 (1995)

[14] Cisneros, C., Martínez-y-Romero, R.P., Núñez-Yépez, H.N., Salas-Brito, A.L.: Limitations on the superposition principle: superselection rules in non-relativistic quantum mechanics. Eur. J. Phys. 19, 237 (1998)

[15] Bartlett, S.D., Rudolph, T., Spekkens, R.W.: Reference frames, superselection rules, and quantum information. Rev. Mod. Phys. 79, 555 (2007)

[16] Hegerfeldt, G.C., Kraus, K., Wigner, E.P.: Proof of the fermion superselection rule without the assumption of time-reversal invariance. J. Math. Phys. 9, 2029 (1968)

[17] Bargman, V.: On unitary ray representations of continuous groups. Ann. Math. 59, 1 (1954)

[18] Gonzalez-Garcia, M.C., Maltoni, M.: Phenomenology with massive neutrinos. Phys. Rep. 460, 1 (2008)

[19] Pontecorvo, B.: Neutrino experiments and the problem of conservation of leptonic charge. Sov. Phys. JETP 26, 984 (1968)

[20] Xu, S.-Y., et al.: Discovery of a Weyl fermion semimetal and topological Fermi arcs. Science 349, 613 (2015)

[21] Lu, L., et al.: Experimental observation of Weyl points. Science 349, 622 (2015)

[22] Xu, S.-Y., et al.: Discovery of a Weyl fermion state with Fermi arcs in niobium arsenide. Nature Physics 11, 748 (2015)

[23] Tsvelik, A.M.: Quantum Field Theory in Condensed Matter Physics, 2nd ed. Cambridge University Press, Cambridge (2003)

[24] Moriya, H.: Some aspects of quantum entanglement for CAR systems. Lett. Math. Phys. 60, 109 (2002)

[25] Araki, H., Lieb, E.H.: Entropy inequalities. Commun. Math. Phys. 18, 160 (1970) 
[26] Friis, N., Lee, A.R., Bruschi, D.E.: Fermionic-mode entanglement in quantum information. Phys. Rev. A 87, 022338 (2013)

[27] Friis, N.: Reasonable fermionic quantum information theories require relativity. New J. Phys. 18, 033014 (2016)

[28] Carlson, B.C., Keller, J.M.: Eigenvalues of density matrices. Phys. Rev. 121, 659 (1961)

[29] Coleman, A.J.: Structure of fermion density matrices. Rev. Mod. Phys. 35, 668 (1963)

[30] Ando, T.: Properties of fermion density matrices. Rev. Mod. Phys. 35, 690 (1963)

[31] Mazziotti, D.A.: Structure of fermionic density matrices: Complete $N$-representability conditions. Phys. Rev. Lett. 108, 263002 (2012)

[32] Borland, R.E., Dennis, K.: The conditions on the onematrix for three-body fermion wavefunctions with onerank equal to six. J. Phys. B 5, 7 (1972)

[33] Schilling, C., Gross, D., Christandl, M.: Pinning of fermionic occupation numbers. Phys. Rev. Lett. 110, 040404 (2013)

[34] Benavides-Riveros, C.L., Springborg, M.: Quasipinning and selection rules for excitations in atoms and molecules. Phys. Rev. A 92, 012512 (2015)

[35] Schilling, C.: Hubbard model: Pinning of occupation numbers and role of symmetries. Phys. Rev. B 92, 155149 (2015)

[36] Cahill, K.E., Glauber, R.J.: Density operators for fermions. Phys. Rev. A 59, 1538 (1999)

[37] Bravyi, S.: Classical capacity of fermionic product channels. ArXiv: quant-ph//0507282

[38] Fannes, M., Van Ryn, N.: Connecting the von Neumann and Rényi entropies for fermions. J. Phys. A: Math. Theor. 45, 385003 (2012)

[39] Sen(De), A., Sen, U., Gromek, B., Bruß, D., Lewenstein, M.: Capacities of quantum channels for massive bosons and fermions. Phys. Rev. Lett. 95, 260503 (2005)

[40] D'Ariano, G.M., Manessi, F., Perinotti, P., Tosini, A.: The Feynman problem and fermionic entanglement: Fermionic theory versus qubit theory. Int. J. Mod. Phys. A 29, 1430025 (2014)

[41] Zimborás, Z., Zeier, R., Keyl, M., Schulte-Herbrüggen, T.: A dynamic systems approach to fermions and their relation to spins. EPJ Quantum Technology 1, 11 (2014)

[42] D'Ariano, G.M., Manessi, F., Perinotti, P., Tosini, A.: Fermionic computation is non-local tomographic and violates monogamy of entanglement. EPL 107, 20009 (2014)

[43] Bravyi, S.B., Kitaev, A.Yu.: Fermionic quantum computation. Ann. Phys. 298, 210 (2002)

[44] Berezin, F.A.: The Method of Second Quantization. Academic Press, New York (1966)

[45] Wiseman, H.M., Vaccaro, J.A.: Entanglement of indistinguishable particles shared between two parties. Phys. Rev. Lett. 91, 097902 (2003)

[46] Evans, D.E.: Completely positive quasi-free maps on the CAR algebra. Commun. Math. Phys. 70, 53 (1979)

[47] Dierckx, B., Fannes, M., Pogorzelska, M.: Fermionic quasifree states and maps in information theory. J. Math.
Phys. 49, 032109 (2008)

[48] Araki, H., Wyss, W.: Representations of canonical anticommutation relations. Helvetica Physica Acta 37, 136 (1964)

[49] Bravyi, S.: Lagrangian representation for fermionic linear optics. Quantum Inf. Comput. 5, 216 (2005)

[50] Greplova, E.: Quantum information with fermionic Gaussian states. Master thesis, University of Munich (2013)

[51] Puspus, X.M., Villegas, K.H., Paraan, F.N.C.: Entanglement spectrum and number fluctuations in the spinpartitioned BCS ground state. Phys. Rev. B 90, 155123 (2014)

[52] Zanardi, P.: Quantum entanglement in fermionic lattices. Phys. Rev. A 65, 042101 (2002)

[53] Amosov, G.G., Mancini, S.: Entanglement from operators splitting. AIP Conf. Proc. 1101, 100 (2009)

[54] Benatti, F., Floreanini, R., Titimbo, K.: Entanglement of identical particles. Open Syst. Inf. Dyn. 21, 1440003 (2014)

[55] Benatti, F., Floreanini, R., Marzolino, U.: Entanglement in fermion systems and quantum metrology. Phys. Rev. A 89, $032326(2014)$

[56] Marzolino, U., Buchleitner, A.: Quantum teleportation with identical particles. Phys. Rev. A 91, 032316 (2015)

[57] Shi, Y.: Quantum entanglement of identical particles. Phys. Rev. A 67, 024301 (2003)

[58] Bañuls, M.-C., Cirac, J.I., Wolf, M.M.: Entanglement in fermionic systems. Phys. Rev. A 76, 022311 (2007)

[59] Iemini, F., Maciel, T.O., Debarba, T., Vianna, R.O.: Quantifying quantum correlations in fermionic systems using witness operators. Quantum Inf. Process. 12, 733 (2013)

[60] Gigena, N., Rossignoli, R.: Entanglement in fermion systems. Phys. Rev. A 92, 042326 (2015)

[61] Boguslawski, K., Tecmer, P., Barcza, G., Legeza, O., Reiher, M.: Orbital entanglement in bond-formation processes. J. Chem. Theory Comput. 9, 2959 (2013)

[62] Rissler, J., Noack, R.M., White, S.R.: Measuring orbital interaction using quantum information theory. Chemical Physics 323, 519 (2006)

[63] Holevo, A.S.: Quantum Systems, Channels, Information, Section 3.1.3. Walter de Gruyter, Berlin (2012)

[64] Lopes, A.A.: Pure univariate quantum marginals and electronic transport properties of geometrically frustrated systems. PhD thesis, Albert Ludwig University of Freiburg (2015)

[65] It is worth mentioning, that particular realizations of $\varrho_{1}$ are known in quantum chemistry as orbital reduced density matrices. In this case, a single orbital corresponds to the algebra $\mathcal{A}_{1}$ generated by operators $a_{\uparrow} a_{\uparrow}^{\dagger}, a_{\uparrow}, a_{\uparrow}^{\dagger}, a_{\uparrow}^{\dagger} a_{\uparrow}$ and $a_{\downarrow} a_{\downarrow}^{\dagger}, a_{\downarrow}, a_{\downarrow}^{\dagger}, a_{\downarrow}^{\dagger} a_{\downarrow}$ corresponding to electron spin projections $+\frac{1}{2}$ and $-\frac{1}{2}$, respectively. In our notation, the 1 -orbital reduced density matrix $\varrho_{1}$ is a two-mode partial state as it takes into account both spin projections. Analogously, 2-orbital reduced density matrix is a four-mode partial state. 